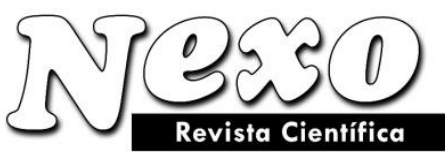

Vol. 33, No. 01, pp. 177-189/Junio 2020

\title{
FACTORES DE ÉXITO EN LAS EMPRESAS DE LA REGIÓN SURESTE DE MÉXICO
}

\section{SUCCESS FACTORS IN COMPANIES IN THE SOUTHEAST REGION OF MEXICO}

\author{
P. E. David Miros*, F. García Landa, M.C David Miros, F. Toto Machucho, \\ J. A. Gómez Pucheta.
}

Tecnológico Nacional de México, Instituto Tecnológico Superior de San Andrés Tuxtla. División de Licenciatura en Administración. San Andrés Tuxtla Veracruz. México.

*paty_david_miros@hotmail.com

\section{RESUMEN}

El éxito de las micro y pequeñas empresas (MyPE) influye de manera positiva en la competitividad y crecimiento económico de una región, de tal manera que la presente investigación se realizó con el objetivo de identificar los factores que inciden en la permanencia de las MyPE del sector terciario en la región sureste de México; caso de estudio región de los TUXTLAS. En este artículo se detalla la metodología utilizada en la investigación utilizando un enfoque cuantitativo y el tipo de estudio descriptivo, para ello se procedió a elaborar un instrumento el cual fue aplicado a los tres principales municipios que integran la región de los Tuxtlas, considerando una población de $4874 \mathrm{MyPE}$, de la cuales se determinó una muestra de 253 micro y pequeñas empresas, por lo que 65 corresponden al municipio de Catemaco, 145 a San Andrés Tuxtla, y 43 a Santiago Tuxtla. Con esta investigación se logró identificar que el control interno, las estrategias de comercialización y la planeación estratégica son aspectos claves para la permanencia de las MyPE en la región Sureste de México.

Palabras clave: Micro y pequeñas empresas; Control interno; Estrategias de comercialización; Planeación estratégica; Permanencia empresarial.

\section{ABSTRACT}

The success of micro and small enterprises (MyPE) positively influences the competitiveness and economic growth of a region, so that this research was conducted with the objective of identifying the factors that affect the permanence of the MyPE of the region. tertiary sector in the southeast region of Mexico; case study of the TUXTLAS region. This article details the methodology used in the research using a quantitative approach and the type of descriptive study, for this we proceeded to develop an instrument which was applied to the three main municipalities that make up the Tuxtlas region, considering a population of 4874 MyPE, of which a sample of 253 micro and small businesses was determined, so 65 correspond to the municipality of Catemaco, 145 to San Andrés Tuxtla, and 43 to Santiago Tuxtla. With this research, it was possible to identify that internal control, marketing strategies and strategic planning are key aspects for the permanence of the MyPE in the Southeast region of Mexico.

Keywords: Micro and small businesses; Internal control; Marketing strategies; Strategic planning; Business permanence. 


\section{P.E. David - Miros et al.}

\section{INTRODUCCIÓN}

En México las micro y pequeñas empresas (MyPE) constituyen el principal motor de la economía nacional ya que ocupan un gran papel en el desarrollo económico del país. De acuerdo con datos de la Encuesta Nacional sobre Productividad y Competitividad de las Micro, Pequeñas y Medianas Empresas (ENAPROCE, 2015) las MyPE representan el 99.6\% de participación dando empleo al $88.9 \%$ del personal siendo las que ocupan una mayor participación en la economía del país.

Al ser México un país donde las MyPE cobran una gran relevancia en la economía, la permanencia de las mismas es dependiente de un mercado tan competitivo e inconstante donde el mínimo descuido podrá afectar la subsistencia de los negocios por lo que según (ENAPROCE, 2015) el 14.7\% de las microempresas manifestaron que no es conveniente el crecimiento de sus negocios y la razón principal por la que no lo desean que estos crezcan se debe a que un $28.4 \%$ microempresas temen ser el blanco fácil de la delincuencia por ello prefieren mantenerse en ese estatus, el $25.1 \%$ se consideran satisfechos con la situación de sus empresas, el $18.6 \%$ lo atribuye a problemas administrativos; y por otro lado el $6 \%$ declaro que los trámites burocráticos son costosos.

De acuerdo a (INEGI, 2016) lo resultados de los nacimientos y muertes de los negocios a nivel nacional en el periodo 2010 - 2015 fueron de 2,225,274 nacimientos y posteriormente de los 4,331,202 de negocios que existían se extinguieron 1, 630, 415 equivalente al 37.6\% del total de establecimientos.

Para (Zapata Guerrero, 2004) en su artículo las pymes y su problemática empresarial las causas se originan tanto en las influencias del entorno como en deficiencias de gestión interna, además que estos problemas no solamente son comunes a las PyMES independiente al sector en el que operen.

(Romero, 2006) realizó una investigación de la competitividad y productividad en empresas familiares PyMES y determinó que en la medida en que las empresas familiares dispongan de reglas y normas de actuación claras y transparentes podrán fortalecer los aspectos que le otorgan competitividad.

De acuerdo a (Velazquez Valdez, 2007) en su investigación las pequeñas y medianas empresas mexicanas requieren aplicar diagnósticos organizacionales la falta o ausencia de planes alternativos o limitarse a un solo plan llevaran a la empresa a no aprovechar las circunstancias y tardar en reaccionar ante los sucesos.

Para (Saenz Vota \& Ávila Arce, 2016) la relación entre la persona con mayor nivel de estudios, influye de manera sustancial en el manejo del negocio y en la productividad del mismo, así como su valor en el mercado, determinando la permanencia en el ámbito laboral.

(Santana , 2017) en su investigación sobre Determinantes de la supervivencia de microempresas en Bogotá: Un análisis con modelos de duración muestra que un $95 \%$ sobrevive el primer año, pero solamente cerca del 50\% llega a los 8 años. La vida promedio de las microempresas canceladas es de 2 a 3 años.

Es conveniente mencionar que algunas empresas fundadas en la región sureste del país hace 20 años o más, han logrado sobrevivir debido a que existía poca competencia al ser la única alternativa para el público, sin embargo en la actualidad esta región ha sido considerada atractiva para la inversión comercial, en mucho se debe a la ubicación geográfica de la zona, esto ha generado el asentamiento de nuevos negocios, lo que pone a la MyPE en desventaja frente a los nuevos negocios debido a que ellos cuentan con una inyección de capital y con estrategias establecidas que les permiten ser competitivas.

Es por ello que la importancia de la investigación radica en que el desarrollo económico de la región de los Tuxtlas, se debe en gran medida al impulso de las MyPE donde predominan los negocios familiares, las cuales han sido heredadas de generación en generación, estas empresas manifiestan que desde sus inicios 


\section{P.E. David - Miros et al.}

han tenido que pasar por varios cambios de tipo social y tecnológicos entre otros, dicha adaptación les ha permitido mantenerse dentro del mercado; entre las herramientas que más destacan podemos mencionar el control interno, la planeación estratégica y las estrategias de comercialización.

El control interno como lo define (Chiavenato I. , 2002) representa el acompañamiento, monitoreo y evaluación del desempeño organizacional para verificar si las tareas se ejecutan de acuerdo a lo planeado, organizado y dirigido.

Así mismo el Informe (Estupiñàn Gaitàn , 2015) define al control interno como un proceso efectuado por el consejo de administración, la dirección y el resto de personal de una entidad, diseñado con el objetivo de proporcionar un grado de seguridad razonable en cuanto a la consecución de objetivos.

Por lo que de esta manera el factor cobra relevancia en la organización permitiendo una retroalimentación de lo ya evaluado, así como el acercamiento hacia las partes de la empresa que de igual manera otorga una mayor participación, verdadero control sobre las mismas y logro de metas y objetivos establecidos.

A su vez las estrategias de comercialización son de gran relevancia para las empresas y sobre todo para aquellas que están en desarrollo ya que se especifican en el mercado meta y una mezcla comercial, en el mercado meta se crean un grupo homogéneo de clientes a los que se deben atraer. En la mezcla comercial son variables controladas en la empresa para satisfacer a los consumidores. (Bencome, Félix. 2012), de manera que, permiten a los empresarios introducirse en el mercado de manera segura y evitar con ello riesgos innecesarios que dañen la producción de la misma.

Inician desde el interior de la organización el movimiento de investigación y desarrollo con un monitoreo constante para identificar oportunidades (Kirchner, 2010).

Igualmente, la planeación estratégica traza la ruta hacia donde se dirige la empresa, establece objetivos estratégicos y financieros, y describe las acciones y enfoques competitivos necesarios para alcanzar los resultados deseados (Thompson, Gamble, Peteraf, \& Strickland III, 2014).

La planeación estratégica, analizada de manera sencilla, es el recurso que ayuda al hombre de negocios a desarrollar habilidades y procedimientos que le permiten lidiar con el futuro (Lerma Kirchner, y otros, 2007).

Es por lo anterior que se plantea la pregunta de investigación: ¿Al determinar los factores permanencia de las micro y pequeñas empresas se podrán formular recomendaciones para fortalecer el desempeño empresarial de los negocios?

De esta manera el documento presenta la metodología aplicada, que incluye el planteamiento del problema, los objetivos generales y específicos, hipótesis con su variable dependiente e independiente; posteriormente se describe el tipo de investigación, se determina la población y muestra, así como la implementación de instrumentos para la recolección de datos. Por último, se presentan los resultados previamente analizados e interpretados para dar pie a la conclusión la cual permitirá determinar cuáles factores inciden en la permanencia de las micro y pequeñas empresas de los Tuxtlas.

\section{MATERIAL Y MÉTODOS}

\subsection{OBJETIVO GENERAL}

Determinar cuáles son los factores de permanencia de las micro y pequeñas empresas en la región de los Tuxtlas, que permitan fortalecer su desempeño empresarial y evitar los riegos que provocan su desaparición.

Nexo Revista Científica / Vol. 33, No. 01, pp. 177-189 / Junio 2020 


\subsection{OBJETIVOS ESPECIFICOS}

- Identificar las micro y pequeñas empresas claves para la investigación.

- Categorizar a las empresas por su giro comercial, para determinar las

- Determinar el promedio de vida de las micro y pequeñas empresas de la región.

- Analizar, determinar y delimitar cuáles son los factores que influyen a la permanencia en las MYPE de la región.

\subsection{HIPÓTESIS}

Los factores que inciden para que una MyPE tenga permanencia en el mercado están relacionados con la implementación de planeación estratégica, control administrativo y al establecimiento de estrategias de comercialización.

Variable dependiente: Permanencia en el mercado.

Variable independiente: Planeación, Control administrativo, estrategias de comercialización.

\subsection{TIPO DE INVESTIGACIÓN}

Esta investigación es cuantitativa, su diseño es no experimental dado que no se busca manipular la variable independiente (Planeación estratégica, control administrativo, estrategias de comercialización), de corte transversal, teniendo en cuenta que la obtención de datos solo se da una vez durante un tiempo determinado. Para fines de la investigación, la muestra es probabilística considerando una población de 4,874 MYPE, de la cual se determinó la muestra de estudio de 253 micro y pequeñas empresas. El tipo de estudio es descriptivo; puesto que se presentan cuáles son algunos de los factores de permanencia en una micro y pequeña empresa de la región de los Tuxtlas y se plantean recomendaciones para mejorar el desempeño empresarial de la región.

\subsection{DETERMINACIÓN DE POBLACIÓN Y MUESTRA}

La población de estudio comprende los municipios de San Andrés Tuxtla, Santiago Tuxtla y Catemaco ubicados en la zona sur del estado de Veracruz, dentro del conjunto montañoso de la sierra de los Tuxtlas. Las actividades económicas de San Andrés Tuxtla se enfocan más en el comercio y a la prestación de servicios como el turismo, mientras que el municipio de Santiago Tuxtla ha destacado por su desarrollo agrícola y ganadero.

Para el municipio de Catemaco, su principal fuente económica es la agricultura, pesca y ganadería; sin embargo, en los últimos años se ha impulsado el desarrollo turístico.

Para determinar la muestra se utilizaron datos de la plataforma de INEGI 2015 en la cual se accedió al servicio de DENUE (Directorio Estadístico Nacional de Unidades Económicas) considerando a la población o universo, con actividad económica de comercio por menor, agricultura, cría y explotación de animales, pesca y caza, información en medios masivos, servicios de apoyo a negocios, servicios de alojamiento temporal y preparación de alimentos y bebidas, servicios inmobiliarios y de alquiler de bienes muebles e intangibles con un tamaño de establecimiento de 0 - 30 personas.

Se utilizó un muestreo probabilístico, mediante los cuales se seleccionaron los negocios de la población que fueron prospectos adecuados para obtener información valiosa para la investigación.

Para determinar la muestra se utilizó la fórmula: 


$$
n=\frac{Z^{2}(p * q)}{N_{e}^{2}+Z^{2}(p * q)}
$$

$\mathrm{N}=$ tamaño de la población

$\mathrm{e}=$ margen de error (porcentaje expresado con decimales)

$\mathrm{z}=$ puntuación $\mathrm{z}$

$\mathrm{p}=$ porcentaje de casos a favor

$\mathrm{q}=$ porcentajes de casos en contra

Sustituimos la formula por los valores:

$\mathrm{z}=1.96$ corresponde a un nivel de confianza de $95 \%$

$\mathrm{p}=50 \%$

$\mathrm{q}=50 \%$

$\mathrm{e}=6 \%$

La muestra resultante fue de 253 micro y pequeñas empresas a las que se le aplicaron las encuestas y los resultados obtenidos se consideraron válidos para toda la población definida.

\subsection{RECOPILACIÓN DE INFORMACIÓN}

Para el diseño del instrumento primero se procedió a su validez, por lo que fue evaluado por 5 especialistas a fin de conocer sus puntos de vista referente a la estructura del documento y que se cumpliera con los requisitos de validez y confiabilidad.

Una vez determinada la muestra y validado el instrumento se aplicaron 253 encuestas, contemplando aspectos como: Género, edad, puesto desempeñado, nombre de la empresa, años de permanencia en el mercado, giro comercial, número de empleados, así como personalidad jurídica. Estos datos se consideraron con el propósito de definir el perfil de los administradores y dueños. Además, el instrumento se conformó por 29 ítems que consideraban cinco dimensiones como: Toma de decisiones, recursos humanos, liderazgo y trabajo en equipo, planeación estratégica, relaciones con proveedores, estrategias de comercialización y control interno mismas que permitieron identificar a los factores de permanencia de la MYPE.

El instrumento se aplicó en los meses de enero febrero de 2019 en los negocios más frecuentados de los tres municipios, ubicados en la zona centro, mercado municipal y zona del boulevard de las tres ciudades.

La tabulación de los datos se hizo en una base de datos y fue validado por tres investigadores con el interés personal de analizar la tendencia de cada uno de los ítems.

\section{RESULTADOS}

Los resultados que se presentan a continuación describen el comportamiento de los micro y pequeños empresarios de las ciudades San Andrés Tuxtla, Santiago Tuxtla y Catemaco en el periodo Enero-febrero 2019; es preciso mencionar que quienes fueron entrevistados, un $48 \%$ son propietarios de los negocios y un $52 \%$ son administradores de los mismos. 
P.E. David - Miros et al.

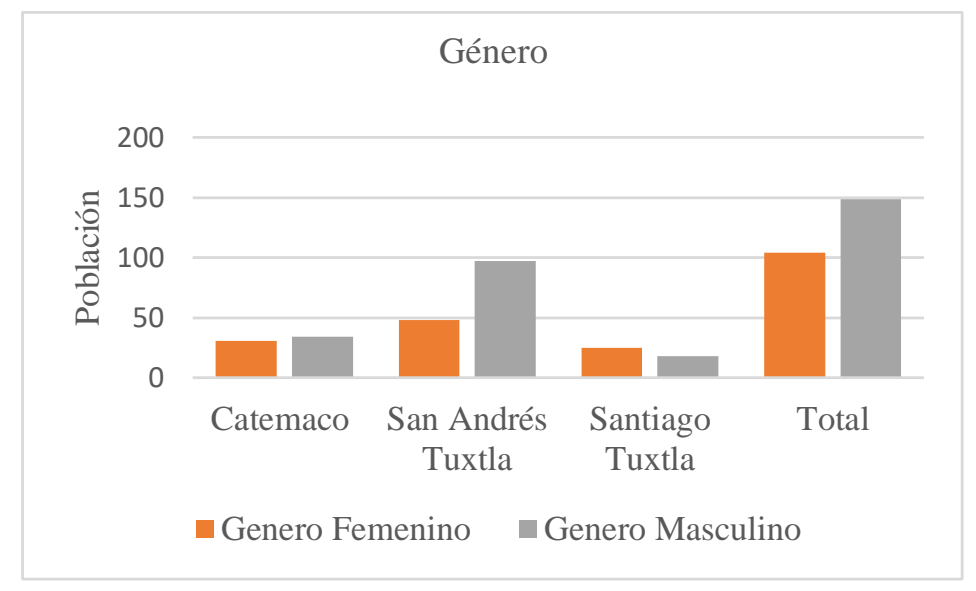

Figura 1. Se aprecia la composición de la muestra en porcentaje de acuerdo al género. Fuente: Elaboración propia.

De acuerdo a los resultados se observa que en los tres municipios la mayoría de los que administran las MyPE son del género masculino con un 58\% y $42 \%$ corresponde al género femenino, lo que refleja que en esta zona el principal proveedor sigue siendo los varones además de que son los que tienen la responsabilidad de dirigir los negocios.

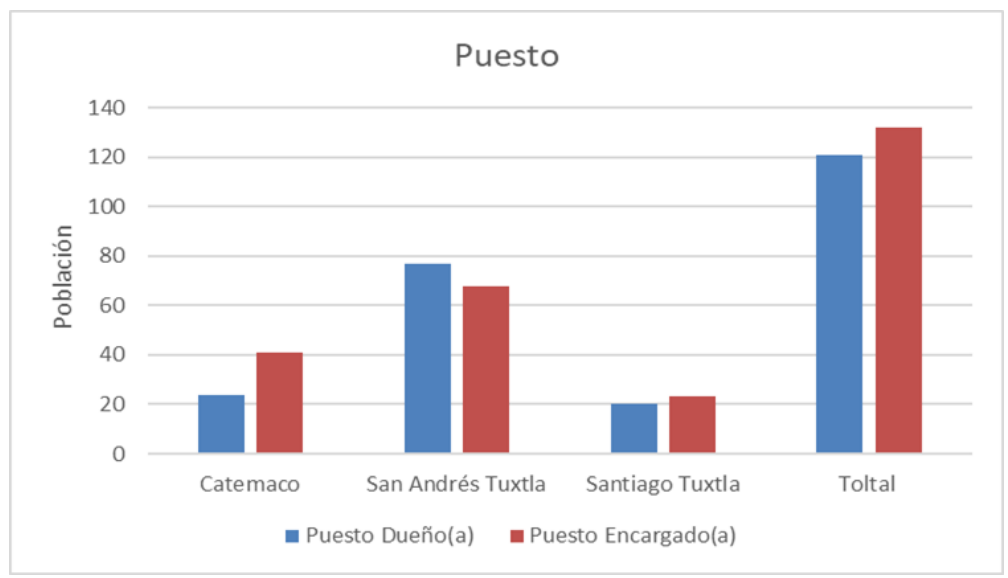

La figura 2. Muestra el perfil que despeña la persona encuestada. Fuente: Elaboración propia

Cómo se puede observar los resultados fueron muy similares y aunque en la mayoría de las MyPE las administre un encargado(a) gran parte de ellos resultan ser familia del dueño(a), lo que permite deducir que la mayoría genera el autoempleo o fuentes de ingresos para sus familiares. 


\section{P.E. David - Miros et al.}

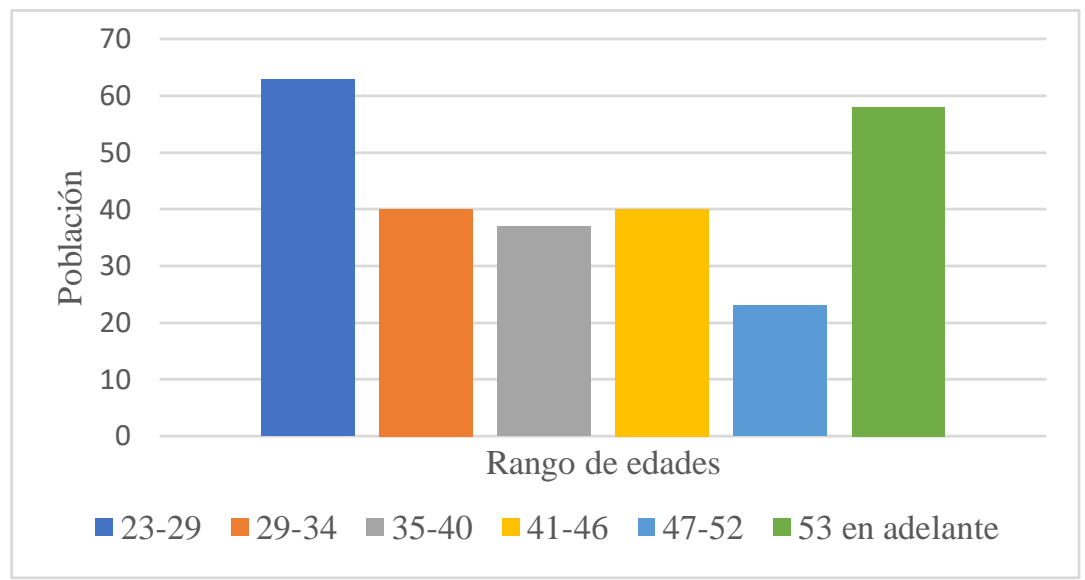

Figura 3. Representa la composición de la muestra según el rango de edades.

Fuente: Elaboración propia

De acuerdo a los resultados se puede observar que la mayoría de los encargados (a) y dueños (a) son en primer lugar personas jóvenes con un rango de edad de 23-29 años, en segundo lugar, se encuentran las personas mayores a 53 años y en un tercer lugar aquellas que se ubican en el rango de 29-46 años.

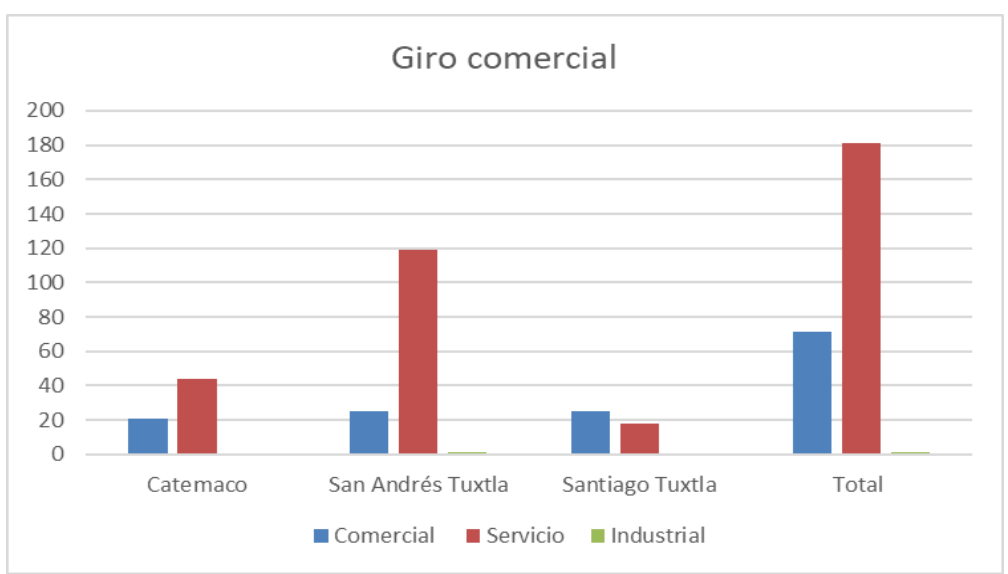

Figura 4. Representa el giro de la empresa Fuente: Elaboración propia

Como lo muestra la figura 4, las MyPE que más predominan son de servicios debido a que la región de los Tuxtlas es considerada una zona turística y la mayoría de los negocios establecidos son hoteles y restaurantes.

La tabla 1. Representa los años de permanencia de una MyPE en el mercado y el factor o factores de éxito que le han permitido permanecer en el mercado.

\begin{tabular}{cc}
\hline Años de permanencia & $\begin{array}{c}\text { Factores de éxito que a las empresas les ha } \\
\text { permitido su permanencia en el mercado. }\end{array}$ \\
\hline $5-9$ & Planeación estratégica y estrategias de \\
comercialización \\
$10-14$ & Estrategias de comercialización \\
$15-19$ & Estrategias de comercialización \\
$20-24$ & Control interno \\
$25-29$ & Estrategias de comercialización \\
$30-34$ & Control interno \\
\hline
\end{tabular}

Nexo Revista Científica / Vol. 33, No. 01, pp. 177-189 / Junio 2020 


\begin{tabular}{cc}
\hline $35-39$ & Control interno \\
40 en adelante & Control interno y planeación estratégica \\
\hline Fuente: Elaboración propia. &
\end{tabular}

El promedio de vida de las MYPE es de 11.26 años, esto indica que la mayoría de los negocios establecidos en la región de los Tuxtlas supera la barrera de los 5 años de vida.

De acuerdo a las encuestas realizadas en la región de los Tuxtlas los factores más relevantes que influyen a la permeancia de las MYPE son el control interno, estrategias de comercialización y planeación estratégica. Con esto se logra comprobar la hipótesis presente investigación.

Tabla 2. Promedio de aspectos obtenidos en relación al control interno.

\begin{tabular}{lc}
\hline \multicolumn{1}{c}{ Aspectos } & Promedio \\
\hline Lleva un control de las operaciones que realiza en el negocio & 4.47 \\
Mantiene altos costos de desperdicios, retrabajos o errores & 2.0 \\
$\begin{array}{l}\text { Posee controles de calidad en la entrega de sus productos o } \\
\text { servicios }\end{array}$ & 4.30 \\
$\begin{array}{l}\text { Evalúa las relaciones con sus proveedores, considerando las } \\
\text { condiciones de entrega de los productos }\end{array}$ & 4.22 \\
$\begin{array}{l}\text { Tiene dificultad para conseguir los insumos o materia prima a } \\
\text { utilizar }\end{array}$ & 1.87 \\
$\begin{array}{l}\text { Diferencia los gastos personales con los gastos del negocio } \\
\text { Tiene un sueldo asignado dentro de la empresa }\end{array}$ & 4.15 \\
\hline Fuente: Elaboración propia. Escala: 1: Nunca, 2: Casi nunca, 3: Ocasionalmente, 4: Casi siempre, 5: Siempre.
\end{tabular}

Es importante destacar que ninguno de los aspectos, en promedio, obtuvo la calificación de dos, asociados con la evaluación de que el control interno siempre es puesto en práctica en dichas MyPE. Solo hay dos aspectos que el promedio se encuentra en 1.87 - 2 del cual tiene relación a que ninguno de los empresarios encuestados casi nunca tiene dificultad para conseguir los insumos o materia prima a utilizar y que de igual manera no mantienen altos costos de desperdicios, retrabajos o errores.

Tabla 3. Promedio de aspectos obtenidos en relación a las estrategias de comercialización.

\begin{tabular}{lc}
\hline \multicolumn{1}{c}{ Aspectos } & Promedio \\
\hline $\begin{array}{l}\text { Aplica estrategias de comercialización, considerando el segmento } \\
\text { de mercado al que está dirigido su negocio }\end{array}$ & 3.86 \\
Reconoce y analiza las estrategias que fija su competencia & 3.67 \\
$\begin{array}{l}\text { Invierte recursos en investigar las tendencias del mercado } \\
\text { Identifica las ventajas competitivas en el mercado }\end{array}$ & 2.51 \\
$\begin{array}{l}\text { Utiliza la publicidad para promocionar sus productos o servicios } \\
\text { Tiene definidos objetivos a corto y largo plazo y los comunica a } \\
\text { sus empleados }\end{array}$ & 3.59 \\
\hline \begin{tabular}{l} 
Fuente: Elaboración propia. Escala: 1: Nunca, 2: Casi nunca, 3: Ocasionalmente, 4: Casi siempre, 5: Siempre. \\
\hline
\end{tabular}
\end{tabular}

Acerca de los aspectos de las estrategias de comercialización se puede observar que la mayoría obtuvieron una calificación 3.5 a 3.9 esto indica que los empresarios están en constante actualización y ponen en práctica dichas estrategias las cueles han favorecido al desarrollo de sus MyPE. Como lo podemos observar en la tabla 3 solo hay un aspecto con un promedio de 2.51 que indica que los empresarios ocasionalmente

Nexo Revista Científica / Vol. 33, No. 01, pp. 177-189 / Junio 2020 


\section{P.E. David - Miros et al.}

invierten en recursos para investigar en las tendencias del mercado y esto se debe a que la mayoría de las empresas mayores a 30 años de permanencia en el mercado no lo creen necesario porque ya tienen su mercado establecido o fidelidad de los clientes. Sin embargo, conviene mencionar que si se puede influir para mejorar el desarrollo de las MyPE con más de 30 años de permanencia poniendo en práctica las estrategias de comercialización ya que las volverá más competentes en el mercado y hasta pueden atraer más clientes.

Tabla 4. Promedio de aspectos obtenidos en relación a la planeación estratégica.

\begin{tabular}{lc}
\hline \multicolumn{1}{c}{ Aspectos } & Promedio \\
\hline $\begin{array}{l}\text { Tiene una visión completa de los recursos de la empresa (humanos, } \\
\text { financieros, técnicos, materiales) }\end{array}$ & 4.1 \\
$\begin{array}{l}\text { Analiza el entorno económico que impacta negativamente en el } \\
\text { negocio }\end{array}$ & 4.03 \\
$\begin{array}{l}\text { Considera que los procesos productivos y de servicios están } \\
\text { definidos considerando los tiempos normales de operación }\end{array}$ & 2.86 \\
\begin{tabular}{l} 
Cuenta con capacidad técnica para contratar a la gente correcta \\
\hline
\end{tabular} & 3.24 \\
\hline Fuente: Elaboración propia. Escala: 1: Nunca, 2: Casi nunca, 3: Ocasionalmente, 4: Casi siempre, 5: Siempre.
\end{tabular}

De acuerdo a los resultados obtenidos, podemos ver que uno de los aspectos que los microempresarios no han considerado aun la medición de los tiempos y el cumplimiento de sus opciones lo que podrían mejorar si se implementan diagramas de procedimientos para mejorar los resultados de sus actividades y mejorar su eficiencia.

Un aspecto interesante en el que podía mejorar es establecer un proceso de selección para contratar al personal, que les permitirá la disminución de costos que implica la contratación de nuevos empleados, así como la constante rotación, por no tener a la gente correcta.

Importante destacar la atención que los microempresarios le prestan al análisis económico, factor que sin duda le ha permitido permanecer en este mercado tan competitivo.

\section{DISCUSIÓN}

Las micro y pequeñas empresas veracruzanas representan un porcentaje considerable en la participación económica del estado. Es por ello que la necesidad de un control interno en la organización surge a raíz de la profesionalización de las mismas debido a un mercado cada vez más demandante y en constante actualización. Puesto que este proceso permite a la empresa medir y evaluar el desempeño de todas sus áreas, así como lograr un orden dentro de las actividades que realizan, es de gran relevancia su aplicación en todo negocio establecido y en aquel que está en crecimiento.

Tal y como lo menciona ( MÜNCH, 2010) aunque una empresa cuente con magníficos planes, una estructura organizacional adecuada y una dirección eficiente, el ejecutivo no podrá verificar cual es la situación real de la organización si no existe un mecanismo que se cerciore e informe si los hechos van de acuerdo con los objetivos.

Así mismo el proceso de control se puede y debe aplicar en todas aquellas actividades que se consideren estratégicas, el número de veces que se considere necesario (Lerma Kirchner, y otros, 2007). 


\section{P.E. David - Miros et al.}

Es importante para todo tipo de organización buscar la implementación del Control Interno de tal forma que facilite a la alta dirección el logro de las metas y los objetivos fijados en contexto con su particular planeación estratégica (Montoya, 2016).

Es de vital trascendencia que el Control Interno forme parte de la estructura de las organizaciones y permita de igual forma a contribuir a alcanzar los objetivos de operación, información y cumplimiento, al mismo tiempo que busque de manera razonable salvaguardar los activos. (Montoya, 2016).

Por lo tanto, la importancia de este factor radica en el hecho de que a pesar que todo en el negocio marche bien si no se implementa un sistema de control, el logro de metas y objetivos será inalcanzable.

También es relevante considerar que la planeación estratégica como lo definen (Lerma Kirchner, y otros, 2007) es el proceso de seleccionar el mejor camino entre dos puntos. El punto 1 es el presente del negocio y el punto 2 su futuro. La planeación estratégica ayuda a seleccionar el mejor punto 2, a elegir los caminos alternativos y a tomar el óptimo para llegar a esa meta.

La planeación, de manera natural, lleva a pensar sobre la esencia de las organizaciones y su destino, esto es, de qué manera habrá de posicionarse en el ambiente, como afrontar los riesgos y oportunidades de ese ambiente; decidir sus ambiciones de largo plazo que habría de traducir en objetivos más específicos a corto plazo, y que medios utilizará para conseguirlo (Torres Hernández, 2014).

Las ventajas competitivas que brinda una planeación estratégica bien aplicada son los conocimientos adquiridos que se traducen en experiencia y habilidades que permiten un buen manejo del negocio, así como en el mercado inconstante al que se enfrentan, de tal manera que en el momento de la elección del camino hacia el futuro se determinara el apropiado para la empresa.

Es por ello que como lo mencionan (Thompson, Gamble, Peteraf, \& Strickland III, 2014) una estrategia clara y razonada es la receta que necesita la gerencia para hacer negocios, su ruta para alcanzar una ventaja competitiva, su plan para complacer a sus clientes y así mejorar su desempeño. Por consiguiente, no cabe duda que la planeación estrategia de una compañía es realmente importante, por lo que los micro y pequeños empresarios tienen la responsabilidad de tomar decisiones las cuales impactaran en la forma de trabajar de sus negocios.

Las estrategias de comercialización es un factor importante en el mundo ya que es la actividad primordial y se realiza cotidianamente en los negocios, comercializar es importante ya que los países tienen una forma de elevar su economía y tener preferencias arancelarias y tratados que puedan tener ventaja competitiva (García Govea \& Concepción Hernández, 2012).

Estas estrategias buscan atraer clientes nuevos cada día, para que con ello se logre la fidelidad por parte de ellos hacia la empresa, una herramienta de esto es conocida como la investigación de mercado la cual permitirá mantener a los clientes ya existentes, es por ello que (Carl McDaniel, 2016) la retención de clientes paga grandes dividendos a las empresas. Propulsados por ventas repetidas y referencias, los ingresos y la participación de mercado crecen. Importancia que radica en el hecho de que implementar estrategias de comercialización permitirán un verdadero posicionamiento y permanencia en el mercado.

Al ser una actividad de gran relevancia para los microempresarios que día a día se realiza no puede descuidarse ya que les permite dentro de un mercado tan demandante dar a conocer sus productos y servicios, así como atraer nuevos clientes y crear fidelidad. 


\section{P.E. David - Miros et al.}

\section{CONCLUSIONES}

A pesar de que los Tuxtlas es una región pequeña en comparación con algunas otras que conforman el estado, la micro y pequeña empresa de servicios y comercial son la principal fuente de empleos y generadoras del desarrollo económico de la misma. Es por ello que en el sector económico de la región representado por los micro y pequeños empresarios tienen la tarea de velar por su permanencia, por consiguiente la identificación del grado de importancia de una cultura empresarial que se ve reflejada en una mejor atención al cliente, una relación más estrecha con sus proveedores, aplicación de estrategias de comercialización correctamente dirigidas al mercado meta, objetivos claramente establecidos, análisis del entorno económico, así como la separación de ingresos y gastos personales han tenido un efecto positivo en los años de vida de las empresas.

Igualmente se logró identificar que la mayoría de empresarios son personas jóvenes y adultos mayores lo cual implica un grado de preparación por parte de los emprendedores jóvenes y experiencia por parte de los adultos mayores donde la aplicación de estos tiene gran relevancia en el momento de realizarse las actividades cotidianas. Entre las recomendaciones dadas por las voces de estos empresarios el tener perseverancia, disciplina y compromiso les han permitido posicionarse en el mercado y ganar la fidelidad de sus clientes a través de los años.

Entre los resultados observados el género femenino ha ido incursionando en la administración de los negocios, sin embargo, aun predomina el género masculino en la dirección de las MyPE, lo que indica que aún se requiere mayor paridad de género dentro de la sociedad Tuxtleca.

En lo que respecta a las edades de los administradores existe un rango mayor de jóvenes millennials que se encuentran laborando en algún establecimiento y los propietarios de los negocios son personas adultas mayores a 29 años de edad.

Por lo tanto, para la fundación de un negocio es necesario estar preparado económicamente e intelectualmente para poder afrontar los retos que el mercado exige y en el caso de no estarlo tener la apertura para invertir en capacitación e identificación de nuevas tendencias.

Con todo ello, el control interno, las estrategias de comercialización y la planeación estratégica, influyen como factor de éxito para las micro y pequeñas empresas sirviendo como herramientas que brindan oportunidades de crecimiento organizacional otorgando a su vez una mayor presencia y permanencia en el mercado, por ello es necesario que las MyPE de la región consideren establecer en toda sus áreas un sistema de control que le permita salvaguardar los recursos de la empresa evitando desvíos de los mismos o la falta del cumplimento de los compromisos con clientes o proveedores evitando perdidas por fraude o negligencia, así como detectar en tiempo posibles desvíos de recursos.

También es importante que los negocios consideren definir con precisión el plan a seguir a fin de poder alcanzar las metas, de otra manera no podrán lograrlo, en ese sentido podrán hacer los ajustes de acuerdo a las circunstancias del entorno.

Por lo que resulta necesario que para lograr la permanencia de una MyPE se debe contar con ventas constantes que generen ingresos así mismo fijar acciones que le permitan un incremento en la participación de mercado, para ello se debe conocer y analizar el comportamiento de su público objetivo a fin ofrecerles productos o servicios acorde a lo que ellos requieren.

Nexo Revista Científica / Vol. 33, No. 01, pp. 177-189 / Junio 2020 


\section{P.E. David - Miros et al.}

\section{BIBLIOGRAFIAS}

MÜNCH, L. (2010). ADMINISTRACIÒN. Gestión organizacional, enfonques y proceso administrativo. MEXICO: Pearson Educaciòn.

Baca, G. (2007). Fundamentos de Ingeniería Económica (4a. ed.). México D.F.: McGraw-Hill.

Carl McDaniel, J. y. (2016). Investigacion de Mercados. En J. y. Carl McDaniel, Investigacion de Mercados. Mexico, D.F.: Cengage Learning.

Chiavenato. (2002). Administración de los nuevos tiempos. MC Graw Hill.

Chiavenato, I. (2002). ADMINISTRACIÓN EN LOS NUEVOS TIEMPOS. Mc Graw Hill.

ENAPROCE. (2015). Encuesta Nacional sobre Productividad y Competitividad de las Micro, Pequeñas Y medianas Empresas. MEXICO: INEGI. Obtenido de https://www.inegi.org.mx/

Estupiñàn Gaitàn , R. (2015). Control interno y fraudes: analisis de informe COSO I, II Y III con base en los ciclos transacciones. Bogotà: Ecoe ediciones .

García Govea, M., \& Concepción Hernández, A. (2012). Estrategias de comercialización.

Garza, J. (2014). Análisis multicriterio de puntos de inflexión de precio en el mercado de divisas. San Nicolás de los Garza, Nuevo León: Universidad Autónoma de Nuevo León.

Gomero, N. (2014). Análisis económico de los impuestos: Impacto en la rentabilidad de las inversiones. Quipukamayoc, 79-87.

INEGI. (2016). Esperanza de vida de los negocios a nivel. MEXICO: INEGI. Obtenido de https://www.inegi.org.mx/ Kirchner, A. E. (2010). Desarrollo de Nuevos Productos, Una Vision Integral. En A. E. Kirchner, Desarrollo de Nuevos Productos, Una Vision Integral (pág. 346). Ciudad de Mexico: CENGAGE Learning.

Lerma Kirchner, A., Martin Granados , M., Castro Martìnez, A., Flores Becerril, M., Martìnez Frìas , H., Ma. del Coral., ... Valdès Heràndez, L. (2007). Liderazgo emprendedor. Còmo ser un emprendedor de èxito y no morir en el intento. Mèxico: Thomson.

Montoya, O. P. (2016). Visión integral del control interno. En O. P. Montoya, Visión integral del control interno. Universidad de Antioquia.

Romero, L. E. (mayo- agosto de 2006). Competitividad y productividad en las empresas familiares pymes. Escuela de Administración de Negocios(57), 131-141.

Rosillo, J. (2005). La inflación: ¿Elemento inocuo en las decisiones de inversión? Forum Empresarial, 20-46. Ruiz, J. (1992). Capital y depreciación: Una aproximación endógena. Valencia, España: Universitat de Valencia. Saenz Vota, C., \& Ávila Arce, A. (enero-junio de 2016). Microempresas y capital humano: un análisis para México. Ra Ximhai(4), 183-195.

Santana , L. (abril-junio de 2017). Determinantes de la supervivencia de microempresas en Bogotá: un análisis con modelos de duración. INNOVAR JOURNAL. Revista de Ciencias Administrativas y Sociales, 27(64), 51-61.

Thompson, A., Gamble, J., Peteraf, M., \& Strickland III, A. (2014). Administraciòn estratègica (Decimonovena edicciòn ed.). Mèxico: Mc Graw Hill.

Torres Hernández, Z. (2014). Administración estratégica (Primera ediciòn ebock ed.). México: Grupo Editorial Patria.

Velazquez Valdez, G. (2007). ¿Las pequeñas y medianas empresas mexicanas requieren aplicar diagnósticos organizacionales? 
Zapata Guerrero, E. E. (septiembre - diciembre de 2004). Las PyMES y su problemática empresarial. Análisis de casos. Escuela de Administración de Negocios(52), 119-135.

\section{DIRECCIONES ELECTRONICAS}

\section{https://www.inegi.org.mx/}

http://www.inegi.org.mx/est/contenidos/proyectos/encuestas/establecimientos/otras/enaproce/default_t.aspx http://www.eumed.net/ce/2012/gme.pdf http://digital.dgb.uanl.mx/la/1080123399/1080123399 MA.PDF http://api.ning.com/files/wTvT3BDmXy03ZNbobj6aQyezHofPKtv1INc9-YFxt*gf**AcypiKj1sLtCBNs2WwBFkRP9RNw90mMTZUKCTdDKt0OBHP*Gb/InformeCosoResumen2.pdf

\section{SEMBLANZA DE LOS AUTORES}

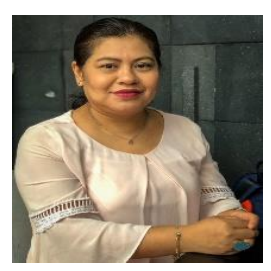

Patricia E. David - Miros: Obtuvo el grado de Licenciada en Administración de Empresas en la Universidad Veracruzana México, Desarrolló sus estudios de Maestría en el Instituto de Investigaciones de las ciencias Administrativas de la Universidad Veracruzana. Se desempeña como docente de tiempo completo en el Instituto Tecnológico Superior de san Andrés Tuxtla, en la División de Licenciatura en Administración, es profesor con perfil deseable. Trabaja en dos líneas de investigación la primera denominada Administración del Desarrollo Empresarial y la segunda Desarrollo de soluciones basadas en tecnologías emergentes. Es integrante del cuerpo académico Ingeniería Administrativa aplicada a proyectos empresariales.

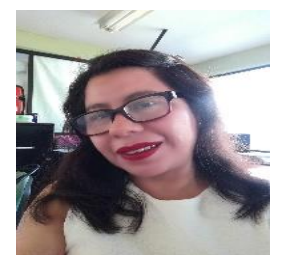

María del C. David-Miros: Obtuvo el grado de Licenciada en Administración de Empresas, y la Maestría en Ciencias Administrativas por parte de la Universidad Veracruzana en Veracruz, México, actualmente se desempeña como profesora con perfil deseable para la carrera de Licenciatura en Administración, en el Instituto Tecnológico Superior de San Andrés Tuxtla, en donde participa en líneas de Investigación Administración del Desarrollo Empresarial; Planeación Empresarial Calidad y Competitividad, es integrante del cuerpo académico Ingeniería Administrativa Aplicada a Proyectos Empresariales,

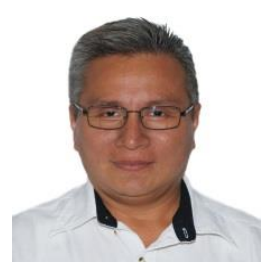

Francisco. Toto-Machucho: obtuvo el grado de Licenciado en Contaduría en el Instituto Tecnológico de Tuxtepec, México; actualmente labora en el Instituto Tecnológico Superior de San Andrés Tuxtla, en la División de Licenciatura en Administración; estudió la maestría en Ciencias Administrativas en la Universidad Veracruzana, México y la especialidad en Competencias Docentes en la Universidad Pedagógica Nacional, México; es líder del cuerpo académico Ingeniería Administrativa Aplicada a Proyectos Empresariales y trabaja en la línea de investigación Administración del Desarrollo Empresarial.

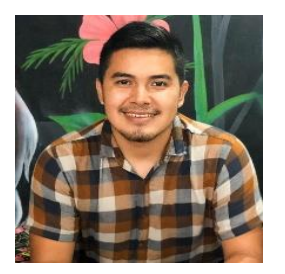

Felipe García -Landa: Estudiante de la Licenciatura en administración en el Instituto Tecnológico Superior de San Andrés Tuxtla. Integrante de Programa Institucional de Semilleros de Investigación.

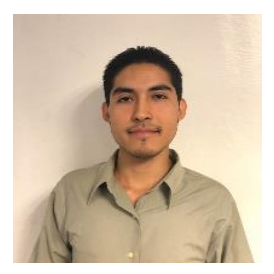

Judá Amisadai Gómez - Pucheta: Estudiante de la Licenciatura en administración en el Instituto Tecnológico Superior de San Andrés Tuxtla. Integrante de Programa Institucional de Semilleros de Investigación. 